Une fois encore je m'excuse de paraître aussi absolu, et, sincèrement, je ne prétends point que tout ce qui précède soit définitif; mais je crois apporter un nouveau moyen d'étude à une question des plus complexes. Il me paraît de nature à permettre de crier "casse-cou " aux conclusionnistes trop pressés; et c'est pourquoi j'ai tenu à en déduire certaines données en partie provisoires sans doute, mais dont l'étude approfondie ne manquera pas de modifier bien des idées fausses, en passe de devenir d'injustifiables dogmes!

\section{USINE HYDRO-ÉLECTRIQUE DE SAUVIAT et Transport de l'Energie à Thiers et à Vichy (Suite et fin)}

\section{LIGNES A HAUTE TENSION}

De l'usine de Sauviat, partent trois lignes à haute tension.

Une première ligne à 11000 volts alimente Courpiére et Thiers, au moyen de 3 fils de $40 \mathrm{~mm}^{2}$. De cette ligne, à l'entrée de Thiers, se dérive celle de St-Rémy qui, en fils de 34/10, dessert les agglomérations de Château-Gaillard, Bellevue, Lombard, Granetias, Le Martinet, La GrandeRoullière, La Monnerie, situées entre Thiers et St-Rémysur-Durolle. De La Momerie, la ligne se continue pour alimenter Chantelauze, Chabreloche, Noirétable et enfin Celle.

Une seconde ligne à 20000 volts, de $20 \mathrm{~mm}^{2}$, alimente St-Dier, Billon, le Moulin-Gardy, Vertaison, etc.

Enfin, une troisième ligne, à 20000 volts, dessert Lezoux, el se prolonge ju'squ'à Vertaison. C'est de celte ligne que part la dérivation de $20 \mathrm{~mm}^{2}$. qui alimente Vichy et dessert sur, son parcours Pont-de-Dore, Puy-Guillaume, Châteldon, et prochainement St-Yorre. Les deux lignes à 20000 volts peuvent se boucler entre elles, et, à l'aide d'interrupteurs de sectionnement, on peut les couper en troncons, de manière à pouvoir exécuter les réparations, sans arrêts pour les clients.

La ligne qui alimente la région de Thiers a $21 \mathrm{kms}$ de longueur jusqu'à Thiers, et 45 jusqu'à Noirćtable. Celle qui dessert vichy est longue de 70 kilomètres.

Toutes ces lignes sont montés presque entièrement sur des poteaux en bois ; toutelois; pour certains parcours, sur des pylônes métalliques. Les poteaux, qui proviennent des magnifiques forêts de sapins de l'Auvergne, ont été préalablement injectés au carbonyle dans un chantier établi à Courpières. Cette injection se fait de la façon suivante: les sapins sont d'abord écorcés, puis séchés pendant un an; une fois parfaitement secs, on les trempe en entier dans une cuve, de 15 mètres de longueur sur 4 mètres de largeur, remplie de carbonyle chauffé à $50^{\circ}$ par de la vapeur. - Les poteaux sont amenés dans la cuve par un pont roulant, et leur immersion se fait au moyen de poids, ce qui permet d'obtenir une certaine pression favorable à l'injection du carbonyle. Après 4 heures d'immersion, les poteaux sont retirés et mis à égouter; deux jours après, on peut les planter. Chaque poteau absorbe $15 \mathrm{kgs}$ de carbonyle, et l'injection revient sensiblement à $3 \mathrm{fr} .50$ par poteau, main d'œuvre comprise.

La portée moyenne des lignes sur poteaux en bois est de $30 \mathrm{~m}$, a vec une flèche de $0^{\mathrm{m}} 40$, pour une température de $15^{\circ}$.

En-dessous des lignes à haute tension est montée une ligne téléphonique dont on a eu soin de croiser les fils tous les $200 \mathrm{~m}$. environ, pour diminuer l'induction dùe aux lignes à haule tension.

Comme cela se fait actuellementsur tous les réseaux, on a supprimé les parafoudres de ligne montés sur poteaux, dont le mauvais fonctionnement, par suite d'un entretien difficile, rend, en général, leur présencé plus dangereuse qu'utile.

Un détail très intéressant à noter, c'est la mise autómatique de la ligne en court-circuit et à la terre, lorsquè l'on manœuvre les interrupteurs de sectionnement des lignes à haute tension. C'est là, en effet, un excellent moyen de sécurité qu'il serait bon de recommander, mais qui cependant ne doit pas faire abandonnor la mise à la terre de la ligne à l'endroit même où l'on travạille. Cette dernière précaution est la seule qui préserve à peu près complètement d'une façon efficace contre les fausses manœuvres.

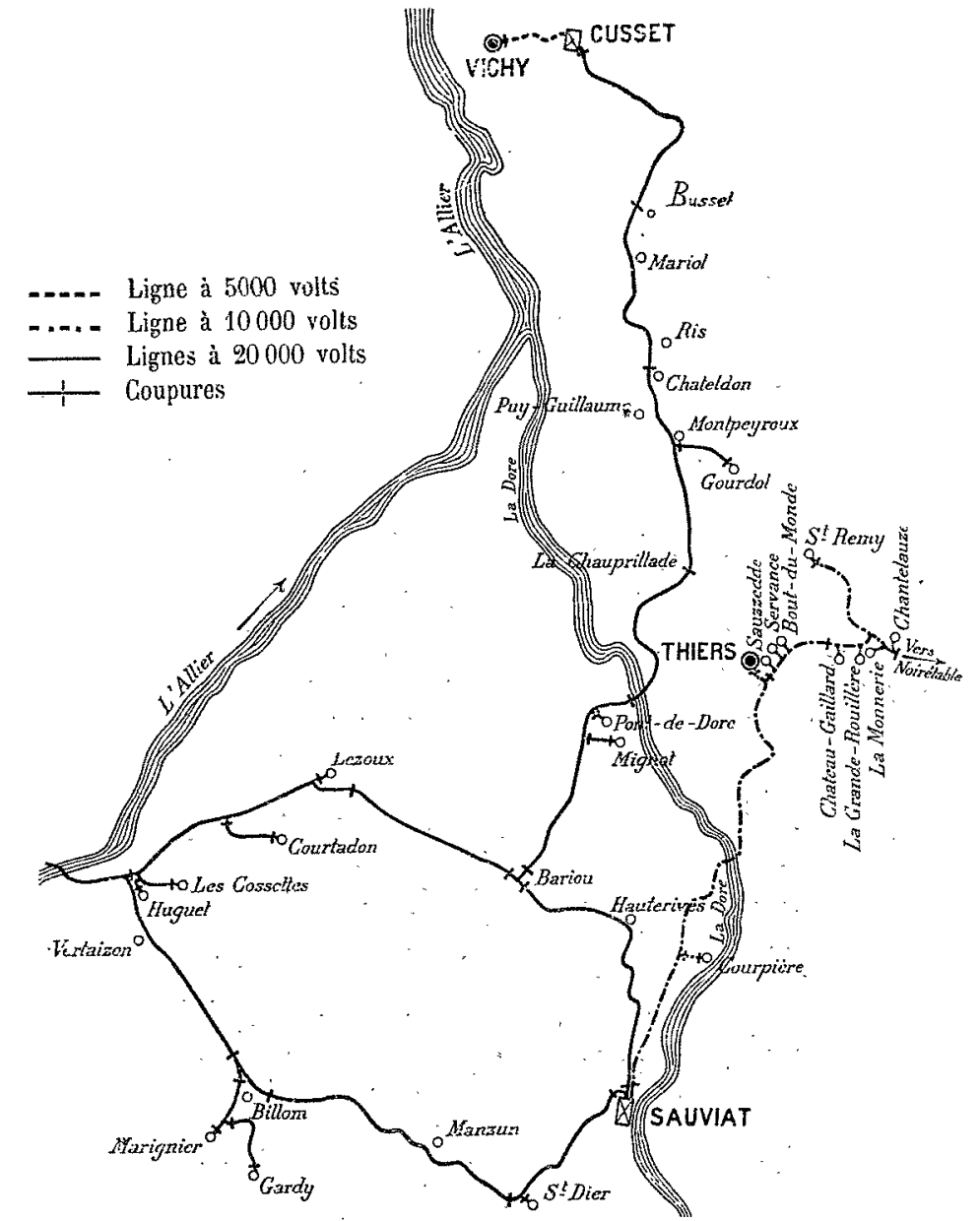

Fig. 13. - Carte du réseau de distribution de la Société des Force Motrices d'Auvergne.

La ligne de Thiers traverse la Dore avec une portée de $130 \mathrm{~m}$. et une flèche de $6 \mathrm{~m}$. Pour cette traversêe, les fils de ligne ont été remplacés par trois cables de $40 \mathrm{~mm}^{2}$, supportés par deux pylônes en fer. Pour la ligne téléphonique on a employé, dans cette traversée, du cable de $12 \mathrm{~mm}^{2}$, avec une flèche de 8 mètres.

La ligne à haute tension qui va jusqu'à Noirétable évite l'agglomération de Thiers, en passant sur la rive gauche de la Durolle, à travers les coteaux desséchés de la Margueride. Pour éviter de faire suivre à la ligne les profondes et nombreuses sinuosités de la montagne, on a établi cette partie de ligne sur pylones en fer avec de très longues portées. Les deux plus grandes atteignent $240 \mathrm{~m}$., avec une flèche de $10 \mathrm{~m}$. Les cables, en bronze silicieux de $16 \mathrm{~mm}^{2}$ de section, sont solidement fixés aux isolateurs au moyen de casçues en bronze, en deux pièces, représentés sur la figure 15 , coiffant les isolateur's et formant serrefils. Dans ces grandes portèes, la distance entre conducteurs est de 1 mètre:

- Réseau de Thiers. - Le réseau à haute tension qui relie les différent postes de transformation répartis dans la 
ville de Thiers est entièrement souterrain. Il est raccordé directement au réseau aérien à 10000 volts à l'intérieur d'un kiosque où sont installés des parafoudres à cornes multiples, des parafoudres à poudre Thury à décharge continue, et de fortes bobines de self. De ce kiosque partent deux lignes souterraines qui peuvent se servir mutuellement de secours.

Le réseau souterrain comprend : $3800 \mathrm{~m}$. de càbles triphasés de $3 \times 20 \mathrm{~mm}^{2}$, et $2300 \mathrm{~m}$. de câbles de $3 \times 10 \mathrm{~mm}^{2}$.

Ce réseau souterrain offre un in térêt tout particulier, car, à l'époque où il fut installé, il n'existait en France, du moins le croyons-nous, que deux villes possédant des câbles triphasés établis pour une tension aussi élevée: Paris (réseau des tramways de l'Est Parisien, $150 \mathrm{kms}$ de construction, à ceux que cette maison a fournis à la Société des Forces Motrices du Rhòne pour le réseau de Lyon á 3300 volts, mais les épaisseurs d'isolant sont plus fortes.

Ils sont formés de trois conducteurs tordus, isolés entre eux et par rapport á la terre par une épaisseur de $9 \mathrm{rm}$. environ de cellulose imprégnée de matière spéciale Berthoud-Borel. Cet isolant est protégé par deux gaines de plomb continues et sans soudures, mises à chaud par la presse hydraulique. Sur la deuxième gaine de plomb sont placés successivement un matelas de filin bien goudronné puis deux rubans de fers feuillards d'un millimètre d'épaisseur chacun, et enfin deux toiles asphaltées.

Tous ces câbles, avant de sorlir de l'usine, ont été soumis

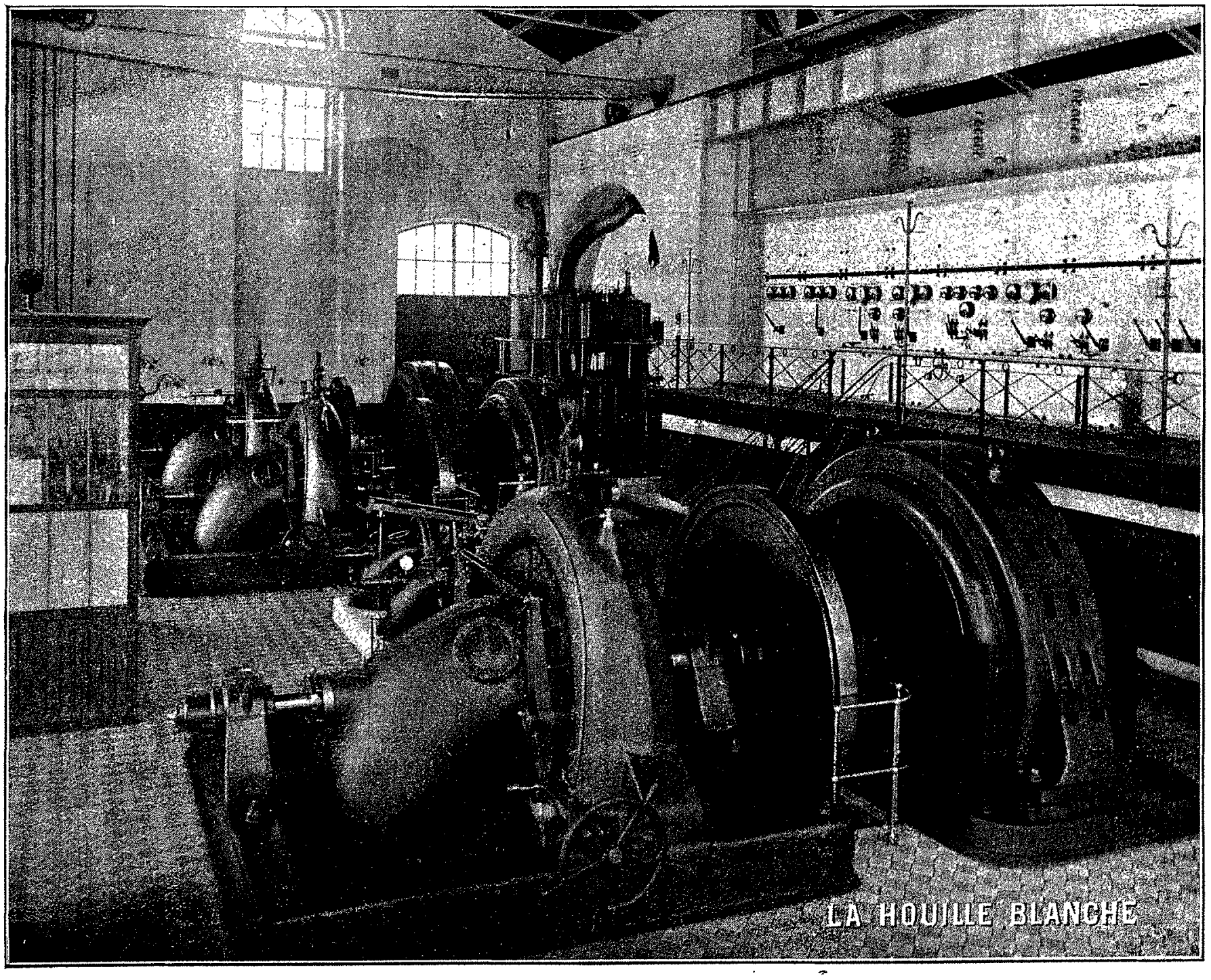

Fa. 14. - Vue intérieure de lusine génératrice de Sauviat.

càbles triphasés à 11000 volts, installés en 1899.1900) et Nice ou la Maison [ierthoud Borel avait également installé, vers 1900, quelques canalisations souterraines triphasées à 11000 volts. Malgré cette tension élevée, les grandes difficultés rencontrées pour la pose des cảbles dans les rues de Thiersqui sont tortueuses, étroites et fort penteuses, et malgré le branchement direct du réseau souterrain sur une longue ligne aérienne, il n'y a pas eu de claquage de câble depuis la mise en marche. Il faut. sans doute, attribuer ce bon fonctionnement à l'excellente fabrication des câbles, mais aussi, certainement aux allernateurs qui, ainsi que nous l'avons dit, fournissent une force électromotrice sensiblement simusoïdale.

Ces câbles, foumis par la Société FraNçaISE dLS CABLES ELECTRIQUES BERTHOUD-BOREL, sont semblables, comme pendant une heure ả des essais de rupture de l'isolant sous la tension alternative efficace de 30000 volts, tant entre conducteurs qu'entre conducteurs et la terre. Pendant quelques secondes, la tension a été poussée jusqu'à 40000 volts. Lisolement kilométrique des câbles à l'usine a été trouvé toujours supérieur à 1000 mégohms.

Après pose, il a été de nouveau procédé á l'essai de claquage des cables en envoyant de l'usine do Sauviat du courant alternatif à la lension efficace de 17500 volts. Ces essais ont été faits tant entre conducteur's qu'entre conducteur's et la terre et chacun d'eux a luré deux heures.

Ces cảbles alimentent douze kiosques en maconnerie, renfermant des transformateurs Alioth, et tous les appa. reils de manceuvre et de protection ordinairement employés. 
Le réseau à basse tension de Thiers, entièrement aérien, fonctionne à la tension de 215 volts composés, avec fit neutre pour permettre l'utilisation pour l'éclairage de la tension étoilée de 125 volts.

Kiosque de transformation de Cusset. - La Société des Forces Motrices d'Auvergne s'est entendue avec la Compagnie du Gaz de Vichy pour lui fournir, à la tension de 5000 volts, le courant dont elle peut avoir besoin pour alimenter ses abonnés. L'usine de Sauviat doit tenir à la disposition de la Compagnie du Gaz une puissance de 500 chevaux, sauf pendant l'été où, par suite du manque d'eau, la fourniture du courant peut être suspendue, la Compagnie du Caz devant produire elle-même le courant qui lui est nécessaire au moyen des groupes électrogènes à vapeur qui alimentaient autrefois son réseau. Le couranl est vendu à la Compagnie du Gaz au compteur d'énergie à un prix assez rémunérateur.

Le kiosque de transformation qui doit abaisser la tension de 20000 à 5000 volts pour alimenter le réseau de Vichy est installé à l'usine à gaz de Cusset.

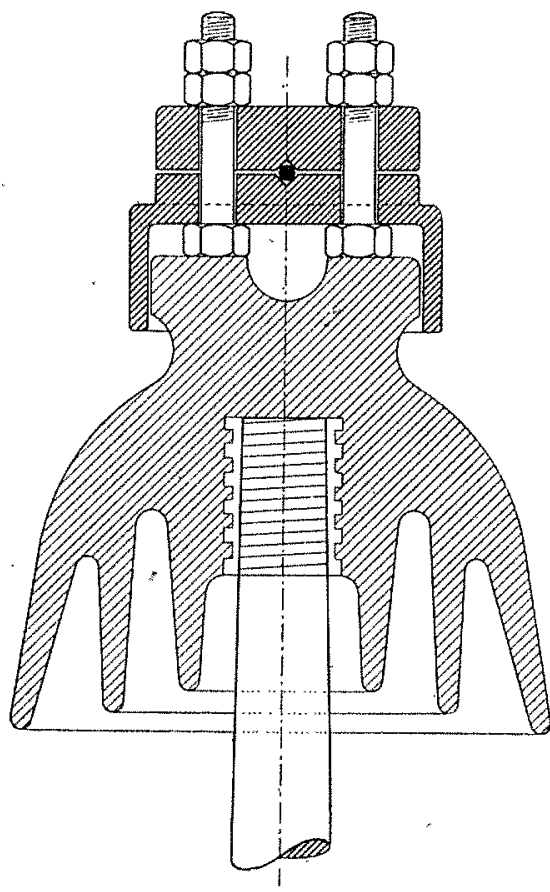

La ligne à 20000 volts pénètre d'abord dans une salle de parafoudres où sont installés des parafoudres à cornes multiples avec résistances liquides, et des bobines de sell. Le courant traverse ensuite des coupe-circuits généraux qui servent plutôt de coupures, car les disjoncteurs de l'usine de Sauviat les remplaçent avantageusement pour cela; il pénètre ensuite dans la seconde partie du bâtiment qui est divisée en deux étages. A l'élage supérieur, sont installés trois transformateurs triphasés, de $300 \mathrm{KVA}$, abaissant la tensión de 20000 à 5000 volts. Ces transformateurs, fournis également par la Société Alioth, sont Fia. $15 .-$ Isolateur pour très
longues portées formateur possédant son à ventilation forcée, chaque transformateur posséda
petit ventilateur actionné par un moteur triphasé.

A l'étage inférieur, se trouvent les tableaux de départ du réseau à 5000 volts, les compteurs d'énergie pour mesurer la consommation de la Compagnie du Gaz, et les tableaux de commande des moteurs des ventilateurs.

\section{EXPLOITATION}

Nous-croyons intéressant d'indiquer ici les différentes manières dont le courant est vendu â Thiers pour la lumière et la force motrice, ainsi que les prix de vente correspondants.

Lumière. - 10 A forfait simple (éclairage de nuit, douilles spèciales).

La lampe de 5 bougies...... 12 franes par an

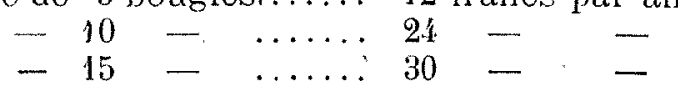

Pour les lámpes avec commutateur, la première se paie 12,24 ou 30 francs, et la seconde, 3 francs.

$2^{\circ}$ A jorfait, au-dessus de 16 bougies, avec limiteur de courant (basculateur JAPY) :
Eclairage de nuit seulement...... $1 \mathrm{fr} .875$ la bougie an Eclairage de jour et de nuit........ 2 î. 500

Le limiteur de courant permet à l'abonné de répartir à son gré le courant pour lequel il est abonné, dans un groupe quelconque de lampes de son installation (*).

$3^{\circ}$ A u compteur horaire (courant de jour et de nuit) :

La lampe de 5 bougies... 1,25 centime par heure

$$
\text { - } 10 \div \ldots 32 \quad \ldots 30-
$$

4. Abonnement spécial pour lampes dateliers, dites "lampes industrielles", et s'allumant du 1er septembre $\grave{a}$ Pâques, avec ou sans basculateur. Pour Thiers seulement.

La lampe de 5 bougies........ 6 francs par an

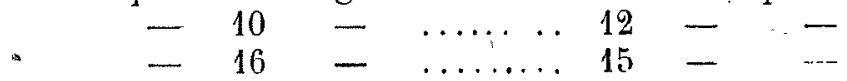

$5^{\circ}$ A u compteur d'énergie (courant de jour et de nuit):

Ateliers............. 0,50 le kilowatt-heure

Magasins........... $0,60 \quad$

Appartements........., 0,65

Prix du compteur horaire............. 36 francs Prix du basculateur posé, arec planchette.... 18 -

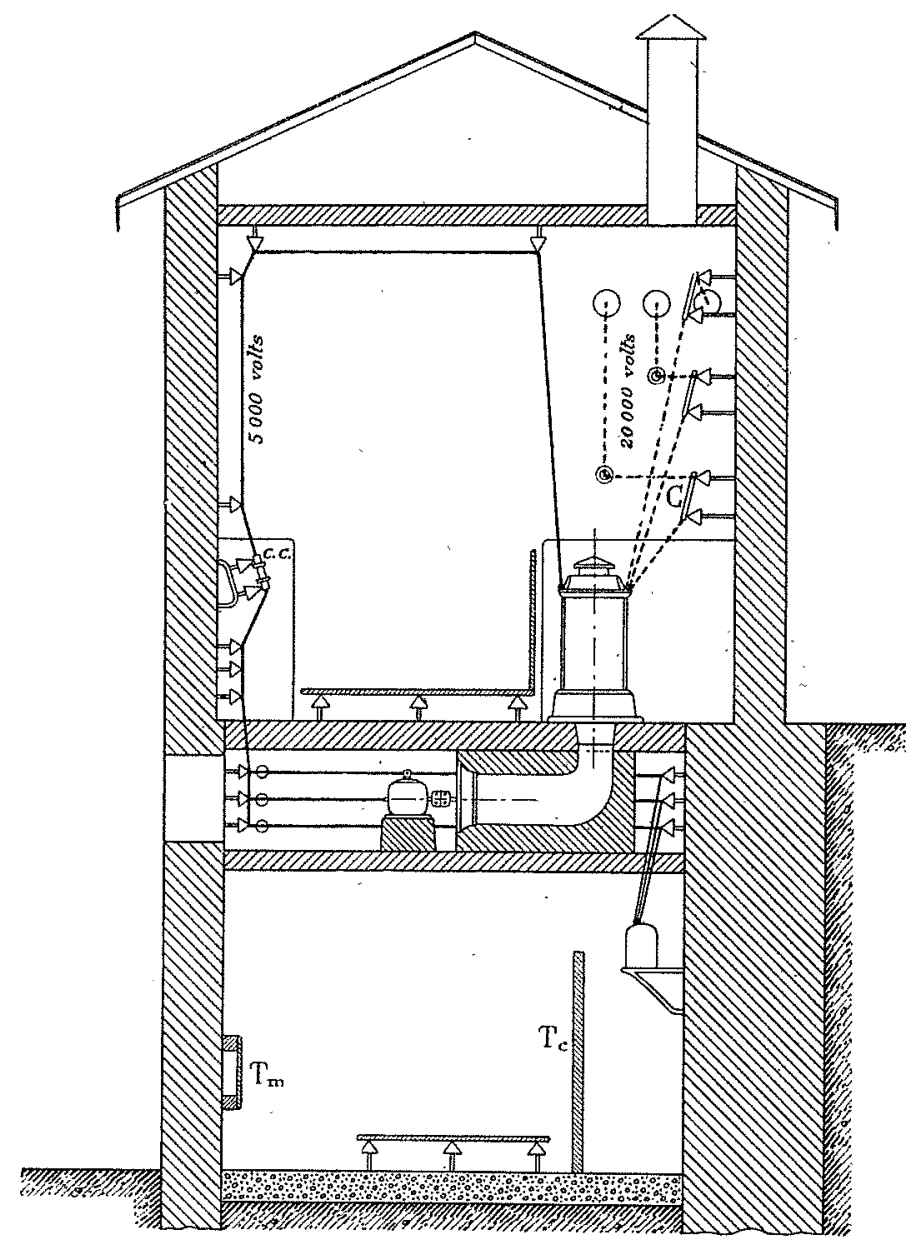

FIG. 16. - Poste de transformation de Cusset.

$\mathrm{T}_{\mathrm{c}}$ Tableau des compteurs

$\mathrm{T}_{\mathrm{m}}$ Tableau des moteurs

cc Coupe-circuits G Coupures

Prix des compteurs, avec tableaux, interrupteur et coupecircuit :

$$
\begin{aligned}
& 5 \text { ampères............... } 86 \text { francs } \\
& 10-\ldots \ldots \ldots \ldots \ldots \ldots \ldots . .69 .
\end{aligned}
$$

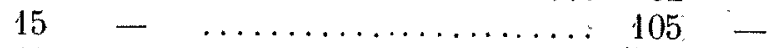

$$
\begin{aligned}
& 30-\ldots \ldots \ldots \ldots \ldots \ldots \ldots \ldots \ldots \ldots \ldots \ldots
\end{aligned}
$$

(*) Le basculateur JAPY, employé ici, a beaucoup d'analogies avec le basculateur ESTRADE dont on trouvera plus loin la deseription: 
Force-motrice. Sans minimum, le kilowatt-heure se paie 0 lr. 30.

Avec minumum :

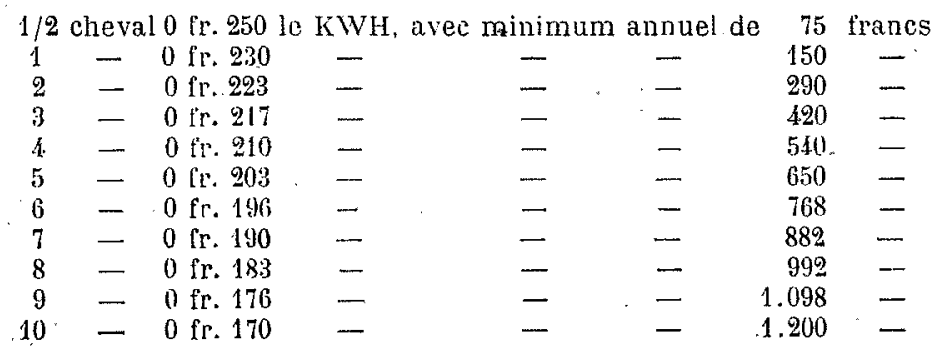

La Société des Forces motrices d'Auvergne fait, en outre, des abonuements spéciaux, à prix très réduits, pour les moteurs destinés à actionner les meules et les polissoirs employés en coutellerie.

A Thiers, où il existe un très grand nombre de petits ateliers de coutellerie, ce sont surtout les moteurs de faible puissance, inférieure à 10 chevaux, qui sont les plus répandus. Ces moteurs rendent de très grands services, comme on peut s'en convaincre en examinant les recettes annuelles assez importantes de la Société, et en établissant, pour chaque année, le nombre relativement très grand des abonnés de force motrice.

On a compté en effet:

Au 31 décembre 1903 (l'usine a été mise en marche le 16. novembre 1903) :

$8.070 \mathrm{fr}$. de recettes.

63 abonnés de force motrice représentant 1381 chevaux.

89 abonnés de lumière représentant 1168 lampes de 10 bougies.

Au 31 décembre 1904:

175.831 franes de recettes.

158 abonnés de force motrice représentant 2210 chevalx

402 abonnés de lumière représentant 5180 lampes de 10 bougies.

Au 31 décembre 1905 :

247.427 francs de receltes.

205 abonnés de force motrice représentant 2345 chevaux.

580 abonnés de lumiere représentant 6908 lampes de 10 bougies.

\section{Au 31 décembre 1906 :}

337329 francs de recettes.

972 abonnés de force motrice représentant 2642 chevaux.

976 abonnés de lumière représentant 9004 lampes de 10 bougies.

Pour l'exercice 1907, qui n'est pas encore clos, la progression des recettes est la même que pour les autres années; ce qui semble assurer un brillaní avenir à la Société des Forces Motrices d'Auvergne.

Cette Société ne se borne pas à développer l'emploi des moteurs, même en dehors de la coutellerie, par de nombreux et intéressants usages (commandes des petites machines à glace, des pétrins mécaniques, etc.), elle cherche également à procurer de nouvelles applications au courant électrique. C'est ainsi qu'elle cherche, en ce moment, un problème difficile, dont elle espère cependant arriver à trouver bientôt la solution pratique, qui consiste à établir un four électrique permettant de porter les lames de couteau à une température bien déterminée (variant suivant la nature de l'acier, ou la destination du couteau, mais voisine de $750^{\circ}$ ) pour pouvoir ensuite les tremper.

Une autre application, plus restreinte, mais assez originale et très intéressante, est celle que le Directeur de la Société a imaginée pour l'aimantation des lames de couteau avec du courant alternatif. Cette aimantation, exigée par certains clients, les Arabes en particulier, qui estiment reconnaître ainsi les lames en bon acier, se fail simplement en plaçant les couteaux à l'intérieur d'un solénoide parcouru par du courant alternatif, et en coupant le courant avec un interrupteur ordinaire. Après linterruption du courant, les couteaux res'ent aimantés. Si J'on veut les désaimanter, il suffit de les replacer dans le solenoïde, et de les retirer sans couper le courant. On peut ainsi (et l'expérience est très curieuse à (aire) aimanter ou désaimanter avec le inême solénoïde, suivant que l'on coupe ou non le courant.

Si, par suite des progrès extraordinairement rapides que l'on a faits ces dernières années, dans l'établissement des grands transports d'énergie électrique, l'installation de la Sociélé des Forces Motrices d'Auvergne n'est plus actuellement très moderne, elle n'en présente pas moins beaucoup d'intérêt, surtout par les dispositions ingénieuses adoptés pour l'aménagement hydraulique, par lo bon agencement de l'installation, qui a toujours très jien fonctionné malgré une tension relativement élevée pour l'époque, surtout pour les alternateurs et les cábles souterrains, et, aussi, malgré l'emploi hardi de très grandes portées poul' une ligne à haute tension, et enfin par les services que l'électricité rend à Thiers, en permettant à ce centre de petite industrie de lutter contre les grandes usines étrangères.

Cette installation fait grand honneur à la Soctété D'AP. PLICATIONS INDUSTRIELLES qui en avait l'entreprise générale et aux Ingénieurs qui l'ont menée à bonne fin : MM. FAY, Berthon et Chapuzot $\left(^{\star}\right)$.

Malheureusement, les débuts de son fonctionnement ont été cruellement attristés par un deuil qui est venu frapper d'une façon brutale et pénible tous ceux qui avaient assisté à la genèse de cette entreprise. Le 5 janvier 1904 , deux mois à peine après l'achèvement de l'œuvre qu'il avait concue avec sa haute intelligence, et qu'il avait fait aboutir à force de travail et d'éneririe, Fay tombait fondroyé dans un kiosque de transformation, a l'age de 28 ans. Coeur d'or, caractère d'une franchise et d'une délicalesse remarquahle, Fay était adoré et estimé de tous ccux qui l'avaient approché ; sa mort a plongé la ville do 'Thiers, ou tout le monde le connaissait, dans une profonde uristesse, je n'en veux pour preuve que les funérailles magnifiques qu'on lui fit, et les larmes sincèes que j’y vis couler. Puisse cette modeste description de son ceuvre honorer sa mémoire, description que, comme son ami, et allié de sa famille, je me suis lait un devoir d'entreprendre, ot que diverses circonstances m'ont empêché de publier plutôt.

\section{P. BERgEON.}

Professeur à linstilut Electrolechnique de Grenolle.

\section{BASCULATEUR ESTRADE}

Pour une usine hydraulique, à qui la puisssance instantanée maxitna importe surtout, le système du tarif à forfait à l'avantage de permettre à l'usine de tirer parti de toute cette puissance. La demande maxima est, en effet, limitée et comme à l'avance. Et l'abonné lui-même est intéressé à limiter le plus possible cette consommation instantanée, puisque d'elle seule dépend le prix de sa fourniture d'électricité.

Dans le cas du tarif à forfait, il importe à l'usine génératrice de s'assurer que ses abonnés ne peuvent frauder, en absorbant, clandestinement, une quantité d'énergie supérieure à celle du forfait. L'appareil suivant, imaginé par M. Estrạde, directeur de la Sociélé méridionale de trans-

(“) Qu'il me soit permis de remereier ici M. Berthon, directeur de la Sociéte Alioth, de lobligeance avec laquello il a bien voulu me fon:mir les elements qui mont mis a memo de faire cet:'o atude, et $\mathrm{M}$. Chapuzo', l'acluel directeur de la Socitete des Forces Motrices d'Auvergno, pour l'amabilité avec laquelle il m'a toujours rega a Thiers, et pour sa complaisance à me renseigner sur son installation. 